\title{
Zeros, Poles, and Fixed Points of Meromorphic Solutions of Difference Painlevé Equations
}

\author{
Shuang-Ting Lan and Zong-Xuan Chen \\ School of Mathematical Sciences, South China Normal University, Guangzhou 510631, China \\ Correspondence should be addressed to Zong-Xuan Chen; chzx@vip.sina.com
}

Received 23 January 2014; Accepted 5 March 2014; Published 6 April 2014

Academic Editor: Zhi-Bo Huang

Copyright (C) 2014 S.-T. Lan and Z.-X. Chen. This is an open access article distributed under the Creative Commons Attribution License, which permits unrestricted use, distribution, and reproduction in any medium, provided the original work is properly cited.

In this paper, we mainly study the properties of transcendental meromorphic solutions $f(z)$ of difference Painlevé equations $w(z+$ 1) $w(z-1)(w(z)-1)=\eta(z) w^{2}(z)-\lambda(z) w(z)$ and $w(z+1) w(z-1)(w(z)-1)=\eta(z) w(z)$ and obtain precise estimations of the exponents of convergence of zeros, poles of $\Delta f(z)$ and $\Delta f(z) / f(z)$, and of fixed points of $f(z+c)$ for any $c \in \mathbb{C}$.

\section{Introduction and Main Results}

At the beginning of last century, Painlevé, Gambier, and Fuchs classified a large number of second-order differential equations in terms of a characteristic which is now known as the Painlevé property [1-4]. Ablowitz et al. [5] considered discrete equations as delay equations in the complex plane which enabled them to utilize complex analytic methods. They looked at, for instance, difference equations of the type

$$
y(z+1)+y(z-1)=R(z, y),
$$

where $R$ is rational in both of its arguments. It is shown that if (1) has at least one nonrational finite-order meromorphic solution, then $\operatorname{deg}_{y} R \leq 2$.

In this paper, we use the basic notions of Nevanlinna's theory (see $[6,7])$. In addition, we use the notations

$\sigma(w)$ to denote the order of growth of the meromorphic function $w(z)$;

$\lambda(w)$ and $\lambda(1 / w)$, respectively, to denote the exponents of convergence of zeros and poles of $w(z)$;

$\tau(w)$ to denote the exponent of convergence of fixed points of $w(z)$.

The quantity $\delta(a, w)$ is called the deficiency of the value $a$ to $w(z)$. Furthermore, we denote by $S(r, w)$ any quantity satisfying $S(r, w)=o(T(r, w))$ for all $r$ outside of a set with finite logarithmic measure and by

$$
\mathcal{S}(w)=\{\alpha \text { meromorphic }: T(r, \alpha)=S(r, w)\}
$$

the field of small functions with respect to $w$. A meromorphic solution $w$ of a difference (or differential) equation is called admissible if all coefficients of the equation are in $\delta(w)$.

Recently, Halburd and Korhonen [8] considered (1) and got the following theorem.

Theorem A. Let $R(z, y)$ be rational in both of its arguments such that its denominator has at least two distinct roots. If the second-order difference equation (1) admits a nonrational meromorphic solution of finite order such that there is a finite real constant $c \geq 1$, such that for sufficiently large $r$,

$$
c^{-1} \bar{n}_{I}(r, w) \leq \bar{n}_{I I}(r, w) \leq c \bar{n}_{I}(r, w)
$$

holds, then (1) is a difference Painlevé II equation

$$
y(z+1)+y(z-1)=\frac{(\lambda z+\mu) y+v}{1-y^{2}},
$$

where $\lambda, \mu$, and $v$ are constants.

Remark 1. If $w$ has a pole at $z=z_{0}$, we say the singularity at $z_{0}$ is of type $I$ if $w\left(z_{0} \pm 1\right)= \pm \varepsilon(\varepsilon= \pm 1)$ and of type $I I$ if $w\left(z_{0} \pm\right.$ $1)=\mp \varepsilon$. We denote by $\bar{n}_{I}(r, w)$ the number of type $I$ poles 
(ignoring multiplicities) in the disc $\{z:|z|<r\}$. Similarly, the function $\bar{n}_{I I}(r, w)$ counts poles of type $I I$.

In 2010, Chen and Shon [9] researched the properties of finite-order meromorphic solutions of difference Painlevé I and II equations. They mainly discussed the existence and the forms of rational solutions and value distribution of transcendental meromorphic solutions. ing.

For difference Painlevé III equations, we recall the follow-

Theorem B (see [10]). Assume that equation

$$
w(z+1) w(z-1)=R(z, w)
$$

has an admissible meromorphic solution $w$ of hyperorder less than one, where $R(z, w)$ is rational and irreducible in $w$ and meromorphic in $z$, then either $w$ satisfies a difference Riccati equation

$$
w(z+1)=\frac{\alpha(z) w(z)+\beta(z)}{w(z)+\gamma(z)},
$$

where $\alpha(z), \beta(z), \gamma(z) \in \mathcal{S}(w)$ are algebroid functions or (5) can be transformed to one of the following equations:

$$
\begin{aligned}
& w(z+1) w(z-1)=\frac{\eta(z) w^{2}(z)-\lambda(z) w(z)+\mu(z)}{(w(z)-1)(w(z)-v(z))} \\
& w(z+1) w(z-1)=\frac{\eta(z) w^{2}(z)-\lambda(z) w(z)}{w(z)-1} \\
& w(z+1) w(z-1)=\frac{\eta(z)(w(z)-\lambda(z))}{w(z)-1} \\
& w(z+1) w(z-1)=h(z) w^{m}(z)
\end{aligned}
$$

In (7a), the coefficients satisfy $\kappa^{2}(z) \mu(z+1) \mu(z-1)=\mu^{2}(z)$, $\lambda(z+1) \mu(z)=\kappa(z) \lambda(z-1) \mu(z+1), \kappa(z) \lambda(z+2) \lambda(z-1)=$ $\kappa(z-1) \lambda(z) \lambda(z+1)$, and one of the following:

(1) $\eta \equiv 1, \nu(z+1) \nu(z-1)=1, \kappa(z)=\nu(z)$;

(2) $\eta(z+1)=\eta(z-1)=\nu(z), \kappa \equiv 1$.

In (7b), $\eta(z) \eta(z+1)=1$ and $\lambda(z+2) \lambda(z-1)=\lambda(z) \lambda(z+1)$. In $(7 \mathrm{c})$, the coefficients satisfy one of the following:

(1) $\eta \equiv 1$ and either $\lambda(z)=\lambda(z+1) \lambda(z-1)$ or $\lambda(z+$ 3) $\lambda(z-3)=\lambda(z+2) \lambda(z-2)$;

(2) $\lambda(z+1) \lambda(z-1)=\lambda(z+2) \lambda(z-2), \eta(z+1) \lambda(z+1)=$ $\lambda(z+2) \eta(z-1)$, and $\eta(z) \eta(z-1)=\eta(z+2) \eta(z-3)$;

(3) $\eta(z+2) \eta(z-2)=\eta(z) \eta(z-1), \lambda(z)=\eta(z-1)$;

(4) $\lambda(z+3) \lambda(z-3)=\lambda(z+2) \lambda(z-2) \lambda(z), \eta(z) \lambda(z)=$ $\eta(z+2) \eta(z-2)$.

In (7d), $h(z) \in \mathcal{S}(w)$ and $m \in \mathbb{Z},|m| \leq 2$.

Zhang and Yang [11] investigated the difference Painlevé III equations (7a)-(7d) with constant coefficients and obtained the following results.
Theorem C. If $w$ is a nonconstant meromorphic solution of difference equation $(7 \mathrm{~d})$, where $m=-2,-1,0,1$ and $h$ is $a$ nonzero constant. Then

(i) $w$ cannot be a rational function;

(ii) $\lambda(w)=\tau(w)=\sigma(w)$.

Lan and Chen [12] studied some difference Painlevé III equations and proved the following.

Theorem D. Suppose that $h(z)$ is a nonconstant rational function. Suppose $w(z)$ is a transcendental meromorphic solution with finite order of $(7 \mathrm{~d})$, where $m=-2,-1,0,1$. Set $\Delta w(z)=$ $w(z+1)-w(z)$. Then

(i) $w(z)$ has no Nevanlinna exceptional value;

(ii) $\lambda(\Delta w)=\lambda(1 / \Delta w)=\sigma(w), \lambda(\Delta w / w)=\lambda(1 /(\Delta w /$ $w))=\sigma(w)$

In general, $\tau(w(z+c)) \neq \tau(w(z))$, where $c$ is a nonzero constant. For example, $w(z)=e^{z}+z, w(z)$ has no fixed points, but $w(z+1)=e e^{z}+z+1$ has infinitely many fixed points and $\tau(w(z+1))=\sigma(w)=1$. Combining Theorems $\mathrm{C}$ and $\mathrm{D}$, we continue to study properties (including fixed points) of transcendental meromorphic solutions of difference Painlevé III equations (7b) and (7c), and obtain the following.

Theorem 2. Suppose that $\eta(z)$ and $\lambda(z)$ are nonconstant polynomials. Suppose $w(z)$ is a transcendental meromorphic solution with finite order of difference Painlevé III equation

$$
w(z+1) w(z-1)(w(z)-1)=\eta(z) w^{2}(z)-\lambda(z) w(z) .
$$

Then

(i) $w(z)$ has at most one Nevanlinna exceptional value;

(ii) for any $c \in \mathbb{C}, w(z+c)$ has infinitely many fixed points, and $\tau(w(z+c))=\sigma(w)$;

(iii) $\lambda(\Delta w)=\lambda(1 / \Delta w)=\sigma(w)$ and $\lambda(1 /(\Delta w / w))=\sigma(w)$;

(iv) if there exists some nonconstant rational function $b(z)$ such that $\lambda(z)=b(z)(\eta(z)-b(z)+1)$, then $\lambda(\Delta w / w)=$ $\sigma(w)$.

Theorem 3. Suppose that $\eta(z)$ is a nonconstant polynomial. Suppose $w(z)$ is a transcendental meromorphic solution with finite order of difference Painlevé III equation

$$
w(z+1) w(z-1)(w(z)-1)=\eta(z) w(z) .
$$

Then

(i) $w(z)$ has no Nevanlinna exceptional value;

(ii) for any $c \in \mathbb{C}, w(z+c)$ has infinitely many fixed points, and $\tau(w(z+c))=\sigma(w)$

(iii) $\lambda(\Delta w)=\lambda(1 / \Delta w)=\sigma(w)$ and $\lambda(1 /(\Delta w / w))=\sigma(w)$;

(iv) if there exists some rational function $b(z)$ such that $\eta(z)=b(z)(b(z)-1)$, then $\lambda(\Delta w / w)=\sigma(w)$. 


\section{Lemmas for the Proofs of Theorems}

Lemma 4 (see [13]). Let $f(z)$ be a meromorphic function of finite order $\sigma$ and let $\eta$ be a nonzero complex number. Then for each $\varepsilon>0$, one has

$$
m\left(r, \frac{f(z+\eta)}{f(z)}\right)+m\left(r, \frac{f(z)}{f(z+\eta)}\right)=O\left(r^{\sigma-1+\varepsilon}\right) .
$$

Lemma 5 (see [13]). Let $f(z)$ be a meromorphic function with order $\sigma=\sigma(f), \sigma<\infty$, and let $\eta$ be a fixed nonzero complex number, then for each $\varepsilon>0$, one has

$$
T(r, f(z+\eta))=T(r, f(z))+O\left(r^{\sigma-1+\varepsilon}\right)+O(\log r) .
$$

Lemmas 4 and 5 show the following.

Lemma 6. Let $c$ be a nonzero constant and $f(z)$ be a meromorphic function with finite order $\sigma$. Then for each $\varepsilon>0$, one has

$$
N\left(r, \frac{1}{f(z+c)}\right)=N\left(r, \frac{1}{f(z)}\right)+O\left(r^{\sigma-1+\varepsilon}\right)+O(\log r) .
$$

Lemma 7 (see $[14,15])$. Let $w$ be a transcendental meromorphic solution of finite order of difference equation

$$
P(z, w)=0,
$$

where $P(z, w)$ is a difference polynomial in $w(z)$. If $P(z, a) \not \equiv 0$ for a meromorphic function $a \in S(w)$, then

$$
m\left(r, \frac{1}{w-a}\right)=S(r, w) .
$$

Lemma 8 (see [15]). Let $f(z)$ be a transcendental meromorphic solution of finite order $\sigma$ of difference equation of the form

$$
U(z, f) P(z, f)=Q(z, f),
$$

where $U(z, f), P(z, f)$, and $Q(z, f)$ are difference polynomials such that the total degree $\operatorname{deg}_{f} U(z, f)=n$ in $f(z)$ and its shifts, and $\operatorname{deg}_{f} Q(z, f) \leq n$. If $U(z, f)$ contains just one term of maximal total degree in $f(z)$ and its shifts, then for each $\varepsilon>0$,

$$
m(r, P(z, f))=O\left(r^{\sigma-1+\varepsilon}\right)+S(r, f)
$$

possibly outside of an exceptional set of finite logarithmic measure.

\section{Proofs of Theorems}

Proof of Theorem 2. (i) By (8), we have

$$
\begin{aligned}
& w(z+1) w(z-1) w(z) \\
& \quad=w(z+1) w(z-1)+\eta(z) w^{2}(z)-\lambda(z) w(z) .
\end{aligned}
$$

Applying Lemma 8 to (17), we have

$$
m(r, w)=S(r, w)
$$

which yields $N(r, w)=T(r, w)+S(r, w)$, that is, $\delta(\infty, w)=0$.
By (17), we have

$$
\frac{\lambda(z)}{w(z)}=\eta(z)+\frac{w(z+1)}{w(z)} \frac{w(z-1)}{w(z)}(1-w(z))
$$

By (18), (19), and Lemma 4, we obtain

$$
\begin{aligned}
m\left(r, \frac{1}{w(z)}\right) \leq & m\left(r, \frac{w(z+1)}{w(z)}\right)+m\left(r, \frac{w(z-1)}{w(z)}\right) \\
& +m(r, w(z))+m(r, \eta(z))+m\left(r, \frac{1}{\lambda(z)}\right) \\
= & S(r, w(z)) .
\end{aligned}
$$

Thus,

$$
N\left(r, \frac{1}{w(z)}\right)=T(r, w(z))+S(r, w(z))
$$

which means that $\delta(0, w)=0$.

Set

$$
\begin{aligned}
P(z, w):= & w(z+1) w(z-1)(w(z)-1) \\
& -\eta(z) w^{2}(z)+\lambda(z) w(z)=0 .
\end{aligned}
$$

Assume that $w(z)$ has two Nevanlinna exceptional values $a, c(a \neq c)$. By $\delta(\infty, w)=0$ and $\delta(0, w)=0$, we see that $a c \neq 0, \infty$. By Lemma 7 , we have $P(z, a) \equiv 0$ and $P(z, c) \equiv 0$. That is,

$$
\begin{gathered}
a^{2}(a-1)-a^{2} \eta(z)+a \lambda(z)=0, \\
c^{2}(c-1)-c^{2} \eta(z)+c \lambda(z)=0 .
\end{gathered}
$$

Hence,

$$
(a-c) \eta(z)=(a-c)(a+c-1) .
$$

Since $a \neq c$, then $\eta(z)=a+c-1$ is a constant. This contradicts the fact that $\eta(z)$ is a nonconstant polynomial. So, $w(z)$ has at most one Nevanlinna exceptional value.

(ii) For any $c \in \mathbb{C}$, substituting $z+c$ for $z$ in (8), we obtain

$$
\begin{aligned}
& w(z+c+1) w(z+c-1)(w(z+c)-1) \\
& \quad=\eta(z+c) w^{2}(z+c)-\lambda(z+c) w(z+c) .
\end{aligned}
$$

Set $g(z)=w(z+c)$. Thus, (25) can be written as

$$
\begin{aligned}
& g(z+1) g(z-1)(g(z)-1) \\
& \quad=\eta(z+c) g(z)^{2}-\lambda(z+c) g(z) .
\end{aligned}
$$

Set

$$
\begin{aligned}
P_{1}(z, g):= & g(z+1) g(z-1)(g(z)-1) \\
& -\eta(z+c) g(z)^{2}+\lambda(z+c) g(z)=0 .
\end{aligned}
$$


Since $\eta(z)$ and $\lambda(z)$ are polynomials,

$$
\begin{aligned}
& P_{1}(z, z) \\
& \quad=z\left(\frac{(z+1)(z-1)^{2}}{z}-z \eta(z+c)+\lambda(z+c)\right) \not \equiv 0 .
\end{aligned}
$$

By $P_{1}(z, z)$ \# 0 and Lemma 7 , we have $m(r, 1 /(g(z)-z))=$ $S(r, g)$, which follows $N(r, 1 /(g(z)-z))=T(r, g)+S(r, g)$. By $g(z)=w(z+c)$ and Lemma 5 , we have $N(r, 1 /(w(z+c)-z))=$ $T(r, w(z))+S(r, w(z))$. Thus, $\tau(w(z+c))=\sigma(w(z))$.

(iii) By (8), we have

$$
\frac{w(z+1)}{w(z)} \frac{w(z-1)}{w(z)}=\frac{\eta(z) w(z)-\lambda(z)}{w(z)(w(z)-1)} .
$$

Applying Valiron-Mohon'ko theorem and Lemma 5 to (29), we obtain

$$
\begin{aligned}
2 T & (r, w(z)) \\
& =T\left(r, \frac{\eta(z) w(z)-\lambda(z)}{w(z)(w(z)-1)}\right)+S(r, w(z)) \\
& =T\left(r, \frac{w(z+1)}{w(z)} \frac{w(z-1)}{w(z)}\right)+S(r, w(z)) \\
& \leq T\left(r, \frac{w(z+1)}{w(z)}\right)+T\left(r, \frac{w(z)}{w(z-1)}\right)+S(r, w(z)) \\
& =2 T\left(r, \frac{w(z+1)}{w(z)}\right)+S\left(r, \frac{w(z+1)}{w(z)}\right)+S(r, w(z)) \\
& \leq 2 T\left(r, \frac{\Delta w(z)}{w(z)}\right)+S(r, w(z)) .
\end{aligned}
$$

Thus,

$$
T(r, w(z)) \leq T\left(r, \frac{\Delta w(z)}{w(z)}\right)+S(r, w(z)) .
$$

By (31) and Lemma 4, we have

$$
\begin{aligned}
N\left(r, \frac{\Delta w}{w}\right) & =T\left(r, \frac{\Delta w}{w}\right)-m\left(r, \frac{\Delta w}{w}\right) \\
& =T\left(r, \frac{\Delta w}{w}\right)+S(r, w) \\
& \geq T(r, w)+S(r, w) .
\end{aligned}
$$

Therefore, $\lambda(1 /(\Delta w / w)) \geq \sigma(w)$, that is, $\lambda(1 /(\Delta w / w))=$ $\sigma(w)$.

Substituting $w(z+1)=w(z)+\Delta w(z), w(z-1)=w(z)-$ $\Delta w(z-1)$ into (8), we see

$$
\begin{gathered}
(w(z)+\Delta w(z))(w(z)-\Delta w(z-1)) \\
=\frac{\eta(z) w^{2}(z)-\lambda(z) w(z)}{w(z)-1},
\end{gathered}
$$

that is,

$$
\begin{gathered}
(\Delta w(z)-\Delta w(z-1)) w(z)-\Delta w(z) \Delta w(z-1) \\
\quad=\frac{-w^{3}(z)+(\eta(z)+1) w^{2}(z)-\lambda(z) w(z)}{w(z)-1} .
\end{gathered}
$$

Let $z_{0}$ be a zero of $w$, by (33), $z_{0}$ is a zero of $w(z)+\Delta w(z)$ or $w(z)-\Delta w(z-1)$. Since $w\left(z_{0}\right)=0$, then $z_{0}$ must be a zero of $\Delta w(z)$ or $\Delta w(z-1)$. Thus, by (21) and Lemma 6, we obtain

$T(r, w(z))$

$$
\begin{aligned}
& =N\left(r, \frac{1}{w(z)}\right)+S(r, w(z)) \\
& \leq N\left(r, \frac{1}{\Delta w(z)}\right)+N\left(r, \frac{1}{\Delta w(z-1)}\right)+S(r, w(z)) \\
& =2 N\left(r, \frac{1}{\Delta w(z)}\right)+S(r, \Delta w(z))+S(r, w(z)) \\
& \leq 2 N\left(r, \frac{1}{\Delta w(z)}\right)+S(r, w(z)) .
\end{aligned}
$$

Hence, $\sigma(w) \leq \lambda(\Delta w)$, that is, $\lambda(\Delta w)=\sigma(w)$.

Applying Valiron-Mohon'ko Theorem and Lemma 5 to (34), we deduce

$3 T(r, w(z))$

$$
\begin{aligned}
= & T\left(r, \frac{-w^{3}(z)+(\eta(z)+1) w^{2}(z)-\lambda(z) w(z)}{w(z)-1}\right) \\
& +S(r, w(z)) \\
= & T(r,(\Delta w(z)-\Delta w(z-1)) w(z)-\Delta w(z) \Delta w(z-1)) \\
& +S(r, w(z)) \\
\leq & T(r, w(z))+2 T(r, \Delta w(z))+2 T(r, \Delta w(z-1)) \\
& +S(r, w(z)) \\
= & T(r, w(z))+4 T(r, \Delta w(z)) \\
& +S(r, \Delta w(z))+S(r, w(z)) \\
\leq & T(r, w(z))+4 T(r, \Delta w(z))+S(r, w(z)) .
\end{aligned}
$$

Thus,

$$
\frac{1}{2} T(r, w(z)) \leq T(r, \Delta w(z))+S(r, w(z)) .
$$

By (18), (37), and Lemma 4, we have

$$
\begin{aligned}
N(r, \Delta w) & =T(r, \Delta w)-m(r, \Delta w) \\
& \geq T(r, \Delta w)-m\left(r, \frac{\Delta w}{w}\right)-m(r, w) \\
& =T(r, \Delta w)+S(r, w) \\
& \geq \frac{1}{2} T(r, w)+S(r, w) .
\end{aligned}
$$

Hence, $\lambda(1 / \Delta w) \geq \sigma(w)$, that is, $\lambda(1 / \Delta w)=\sigma(w)$. 
(iv) Suppose that $\lambda(z)=b(z)(\eta(z)-b(z)+1)$, where $b(z)$ is some nonconstant rational function. Now we prove that $\lambda(\Delta w / w)=\sigma(w)$. Set

$$
G(z)=(\Delta w(z)-\Delta w(z-1)) w(z)-\Delta w(z) \Delta w(z-1) .
$$

By (34), (39), and $\lambda(z)=b(z)(\eta(z)-b(z)+1)$, we have

$$
\begin{aligned}
G(z) & =\frac{-w^{3}(z)+(\eta(z)+1) w^{2}(z)-\lambda(z) w(z)}{w(z)-1} \\
& =\frac{-w(z)(w(z)-b(z))(w(z)-\eta(z)+b(z)-1)}{w(z)-1} .
\end{aligned}
$$

Since $b(z)$ is a nonconstant rational function, then $b(z+$ $1) / b(z) \not \equiv b(z) / b(z-1)$. By $(22)$ and $\lambda(z)=b(z)(\eta(z)-b(z)+$ 1), we know

$$
\begin{aligned}
P(z, & b(z)) \\
= & b(z+1) b(z-1)(b(z)-1) \\
& -b^{2}(z) \eta(z)+b(z) \lambda(z) \\
= & b(z+1) b(z-1)(b(z)-1)-b^{2}(z) \eta(z) \\
& +b^{2}(z)(\eta(z)-b(z)+1) \\
= & b^{2}(z)(b(z)-1)\left(\frac{b(z+1)}{b(z)} \frac{b(z-1)}{b(z)}-1\right) \neq \equiv 0 .
\end{aligned}
$$

Similarly, we obtain $P(z, \eta(z)-b(z)+1) \not \equiv 0$. By $P(z, b(z)) \not \equiv$ $0, P(z, \eta(z)-b(z)+1) \not \equiv 0$ and Lemma 7 , we have

$$
\begin{gathered}
m\left(r, \frac{1}{w-b(z)}\right)=S(r, w), \\
m\left(r, \frac{1}{w-\eta(z)+b(z)-1}\right)=S(r, w) .
\end{gathered}
$$

By (18), (20), (40) and (42), we obtain

$$
\begin{aligned}
m\left(r, \frac{1}{G}\right) \leq & m\left(r, \frac{1}{w-b}\right)+m\left(r, \frac{1}{w-\eta+b-1}\right) \\
& +m(r, w)+m\left(r, \frac{1}{w}\right) \\
= & S(r, w) .
\end{aligned}
$$

By (39), we obtain

$$
\frac{G(z)}{\Delta w(z) \Delta w(z-1)}=\frac{1}{\Delta w(z)}\left(\frac{\Delta w(z)}{\Delta w(z-1)}-1\right) w(z)-1
$$

It sees from Lemma 4 that

$$
\begin{aligned}
& m\left(r, \frac{1}{\Delta w(z) \Delta w(z-1)}\right) \\
& \quad \leq m\left(r, \frac{1}{(\Delta w(z))^{2}}\right)+m\left(r, \frac{\Delta w(z)}{\Delta w(z-1)}\right) \\
& \quad=2 m\left(r, \frac{1}{\Delta w(z)}\right)+S(r, \Delta w(z)), \\
& 2 m\left(r, \frac{1}{\Delta w(z)}\right) \\
& \quad=m\left(r, \frac{1}{(\Delta w(z))^{2}}\right) \\
& \quad \leq m\left(r, \frac{1}{\Delta w(z) \Delta w(z-1)}\right)+m\left(r, \frac{\Delta w(z-1)}{\Delta w(z)}\right) \\
& \quad=m\left(r, \frac{1}{\Delta w(z) \Delta w(z-1)}\right)+S(r, \Delta w(z)) .
\end{aligned}
$$

Hence,

$$
\begin{aligned}
& m\left(r, \frac{1}{\Delta w(z) \Delta w(z-1)}\right) \\
& \quad=2 m\left(r, \frac{1}{\Delta w(z)}\right)+S(r, \Delta w(z)) .
\end{aligned}
$$

From (18), (43), (44), (46) and Lemma 4, we deduce that

$$
\begin{aligned}
& 2 m\left(r, \frac{1}{\Delta w(z)}\right) \\
&=m\left(r, \frac{1}{\Delta w(z) \Delta w(z-1)}\right)+S(r, \Delta w(z)) \\
& \leq m\left(r, \frac{1}{G(z)}\right)+m\left(r, \frac{1}{\Delta w(z)}\right)+m\left(r, \frac{\Delta w(z)}{\Delta w(z-1)}\right) \\
&+m(r, w(z))+S(r, \Delta w(z)) \\
& \leq m\left(r, \frac{1}{\Delta w(z)}\right)+S(r, \Delta w(z))+S(r, w(z)) \\
& \leq m\left(r, \frac{1}{\Delta w(z)}\right)+S(r, w(z)),
\end{aligned}
$$

which yields

$$
m\left(r, \frac{1}{\Delta w(z)}\right)=S(r, w(z))
$$

By (18) and (48) we have

$$
\begin{aligned}
m\left(r, \frac{1}{\Delta w / w}\right)= & m\left(r, \frac{w}{\Delta w}\right) \leq m(r, w) \\
& +m\left(r, \frac{1}{\Delta w}\right)=S(r, w),
\end{aligned}
$$


and by (31),

$$
N\left(r, \frac{1}{\Delta w / w}\right)=T\left(r, \frac{\Delta w}{w}\right)+S(r, w) \geq T(r, w)+S(r, w) .
$$

Then $\lambda(\Delta w / w) \geq \sigma(w)$. So, $\lambda(\Delta w / w)=\sigma(w)$.

Proof of Theorem 3. (i) By (9), we have

$$
\begin{aligned}
& w(z+1) w(z-1) w(z) \\
& \quad=w(z+1) w(z-1)+\eta(z) w(z) .
\end{aligned}
$$

Applying Lemma 8 to (51), we have

$$
m(r, w)=S(r, w)
$$

Thus, $N(r, w)=T(r, w)+S(r, w)$, which yields $\delta(\infty, w)=0$.

Again by (9), we have

$$
\frac{\eta(z)}{w(z)}=\frac{w(z+1)}{w(z)} \frac{w(z-1)}{w(z)}(w(z)-1) .
$$

From (52), (53), and Lemma 4, we deduce that

$$
\begin{aligned}
m\left(r, \frac{1}{w}\right) \leq & m\left(r, \frac{w(z+1)}{w(z)}\right)+m\left(r, \frac{w(z-1)}{w(z)}\right) \\
& +m(r, w)+m\left(r, \frac{1}{\eta}\right) \\
= & S(r, w),
\end{aligned}
$$

which follows

$$
N\left(r, \frac{1}{w(z)}\right)=T(r, w(z))+S(r, w) .
$$

Thus, $\delta(0, w)=0$.

Set

$$
P(z, w):=w(z+1) w(z-1)(w(z)-1)-\eta(z) w(z) .
$$

For any $a \in \mathbb{C} \backslash\{0\}$, since $\eta(z)$ is a nonconstant polynomial, then $P(z, a)=a^{2}(a-1)-a \eta(z) \not \equiv 0$. By $P(z, a) \not \equiv 0$ and Lemma 7, we know that $m(r, 1 /(w-a))=S(r, w)$, which means that $N(r, 1 /(w-a))=T(r, w)+S(r, w)$. Hence, $\delta(a, w)=0$. Combining $\delta(\infty, w)=0, \delta(0, w)=0$, we see that $w$ has no Nevanlinna exceptional value. that

(ii) For any $c \in \mathbb{C}$, substituting $z+c$ for $z$ in (9), we see

$$
\begin{aligned}
& w(z+c+1) w(z+c-1)(w(z+c)-1) \\
& \quad=\eta(z+c) w(z+c) .
\end{aligned}
$$

Set $g(z)=w(z+c)$. Thus, (57) can be written as

$$
g(z+1) g(z-1)(g(z)-1)=\eta(z+c) g(z) .
$$

Set

$$
\begin{aligned}
P_{1}(z, g):= & g(z+1) g(z-1)(g(z)-1) \\
& -\eta(z+c) g(z)=0 .
\end{aligned}
$$

Since $\eta(z)$ is a polynomial, then

$$
P_{1}(z, z)=z\left(\frac{(z+1)(z-1)^{2}}{z}-\eta(z+c)\right) \not \equiv 0 .
$$

By this and Lemma 7, we have $m(r, 1 /(g(z)-z))=S(r, g)$, which follows $N(r, 1 /(g(z)-z))=T(r, g(z))+S(r, g(z))$. By $g(z)=w(z+c)$ and Lemma 5 , we see that $N(r, 1 /(w(z+c)-$ $z))=T(r, w(z))+S(r, w(z))$. Thus, $\tau(w(z+c))=\sigma(w(z))$.

(iii) Substituting $w(z+1)=w(z)+\Delta w(z), w(z-1)=$ $w(z)-\Delta w(z-1)$ into (9), we have

$$
(w(z)+\Delta w(z))(w(z)-\Delta w(z-1))=\frac{\eta(z) w(z)}{w(z)-1} .
$$

If $z_{0}$ is a zero of $w(z)$, by (61), $z_{0}$ must be a zero of $\Delta w(z)$ or $\Delta w(z-1)$. Thus, by (55) and Lemma 6, we have

$$
\begin{aligned}
T & (r, w(z)) \\
& =N\left(r, \frac{1}{w(z)}\right)+S(r, w(z)) \\
& \leq N\left(r, \frac{1}{\Delta w(z)}\right)+N\left(r, \frac{1}{\Delta w(z-1)}\right)+S(r, w(z)) \\
& \leq 2 N\left(r, \frac{1}{\Delta w(z)}\right)+S(r, w(z)) .
\end{aligned}
$$

Hence, $\sigma(w) \leq \lambda(\Delta w)$, that is, $\lambda(\Delta w)=\sigma(w)$.

By (61), we have

$$
\begin{aligned}
& (\Delta w(z)-\Delta w(z-1)) w(z)-\Delta w(z) \Delta w(z-1) \\
& \quad=\frac{-w^{3}(z)+w^{2}(z)+\eta(z) w(z)}{w(z)-1} .
\end{aligned}
$$

Applying Valiron-Mohon'ko theorem and Lemma 5 to (63), we deduce

$$
\begin{aligned}
3 T( & , w(z)) \\
= & T\left(r, \frac{-w^{3}(z)+w^{2}(z)+\eta(z) w(z)}{w(z)-1}\right)+S(r, w(z)) \\
= & T(r,(\Delta w(z)-\Delta w(z-1)) w(z)-\Delta w(z) \Delta w(z-1)) \\
& +S(r, w(z)) \\
\leq & T(r, w(z))+2 T(r, \Delta w(z)) \\
& +2 T(r, \Delta w(z-1))+S(r, w(z)) \\
= & T(r, w(z))+4 T(r, \Delta w(z)) \\
& +S(r, \Delta w(z))+S(r, w(z)) \\
\leq & T(r, w(z))+4 T(r, \Delta w(z))+S(r, w(z)) .
\end{aligned}
$$


Hence,

$$
\frac{1}{2} T(r, w(z)) \leq T(r, \Delta w(z))+S(r, w(z)) .
$$

Combining (65) with (52) and Lemma 4, we have

$$
\begin{aligned}
N(r, \Delta w) & =T(r, \Delta w)-m(r, \Delta w) \\
& \geq T(r, \Delta w)-m\left(r, \frac{\Delta w}{w}\right)-m(r, w) \\
& =T(r, \Delta w)+S(r, w) \\
& \geq \frac{1}{2} T(r, w)+S(r, w),
\end{aligned}
$$

which yields $\lambda(1 / \Delta w) \geq \sigma(w)$. So, $\lambda(1 / \Delta w)=\sigma(w)$.

By (9), we have

$$
\frac{w(z+1)}{w(z)} \frac{w(z-1)}{w(z)}=\frac{\eta(z)}{w(z)(w(z)-1)} .
$$

Applying Valiron-Mohon'ko theorem and Lemma 5 to (67), we obtain

$$
\begin{aligned}
2 T & (r, w(z)) \\
& =T\left(r, \frac{\eta(z)}{w(z)(w(z)-1)}\right)+S(r, w(z)) \\
& =T\left(r, \frac{w(z+1)}{w(z)} \frac{w(z-1)}{w(z)}\right)+S(r, w(z)) \\
& \leq T\left(r, \frac{w(z+1)}{w(z)}\right)+T\left(r, \frac{w(z)}{w(z-1)}\right)+S(r, w(z)) \\
& =2 T\left(r, \frac{w(z+1)}{w(z)}\right)+S\left(r, \frac{w(z+1)}{w(z)}\right)+S(r, w(z)) \\
& \leq 2 T\left(r, \frac{\Delta w(z)}{w(z)}\right)+S(r, w(z)) .
\end{aligned}
$$

Thus,

$$
T(r, w(z)) \leq T\left(r, \frac{\Delta w(z)}{w(z)}\right)+S(r, w(z)) .
$$

By (69) and Lemma 4, we see that

$$
\begin{aligned}
N\left(r, \frac{\Delta w}{w}\right) & =T\left(r, \frac{\Delta w}{w}\right)-m\left(r, \frac{\Delta w}{w}\right) \\
& =T\left(r, \frac{\Delta w}{w}\right)+S(r, w) \\
& \geq T(r, w)+S(r, w) .
\end{aligned}
$$

Hence, $\lambda(1 /(\Delta w / w)) \geq \sigma(w)$, that is, $\lambda(1 /(\Delta w / w))=\sigma(w)$.

(iv) Suppose that $\eta(z)=b(z)(b(z)-1)$, where $b(z)$ is some rational function. Now we prove that $\lambda(\Delta w / w)=\sigma(w)$. Set

$$
G(z)=(\Delta w(z)-\Delta w(z-1)) w(z)-\Delta w(z) \Delta w(z-1) .
$$

By (63), (71), and $\eta(z)=b(z)(b(z)-1)$, we have

$$
\begin{aligned}
G(z) & =\frac{-w(z)\left(w^{2}(z)-w(z)-\eta(z)\right)}{w(z)-1} \\
& =\frac{-w(z)(w(z)-b(z))(w(z)+b(z)-1)}{w(z)-1} .
\end{aligned}
$$

Since $\eta(z)=b(z)(b(z)-1)$ is a nonconstant polynomial, $b(z)$ is a nonconstant rational function. Then $b(z+1) / b(z) \neq \equiv$ $b(z) / b(z-1)$. By (56) and $\eta(z)=b(z)(b(z)-1)$, we know

$$
\begin{aligned}
& P(z, b(z)) \\
& \quad=b^{2}(z)(b(z)-1)\left(\frac{b(z+1)}{b(z)} \frac{b(z-1)}{b(z)}-1\right) \not \equiv 0 .
\end{aligned}
$$

Similarly, we obtain $P(z,-b(z)+1) \not \equiv 0$. By $P(z, b(z)) \not \equiv 0$, $P(z,-b(z)+1) \neq \equiv 0$, and Lemma 7, we have

$$
\begin{aligned}
m\left(r, \frac{1}{w(z)-b(z)}\right) & =S(r, w(z)), \\
m\left(r, \frac{1}{w(z)+b(z)-1}\right) & =S(r, w(z)) .
\end{aligned}
$$

'By (52), (54), (74), and (72), we obtain

$$
\begin{aligned}
m\left(r, \frac{1}{G}\right) \leq & m(r, w)+m\left(r, \frac{1}{w}\right)+m\left(r, \frac{1}{w-b}\right) \\
& +m\left(r, \frac{1}{w+b-1}\right)=S(r, w)
\end{aligned}
$$

Using the same method as in the proof of (iv) in Theorem 2, we may obtain $m(r, 1 / \Delta w)=S(r, w)$. By this and (52), we have

$$
\begin{aligned}
m\left(r, \frac{1}{\Delta w / w}\right) & =m\left(r, \frac{w}{\Delta w}\right) \leq m(r, w)+m\left(r, \frac{1}{\Delta w}\right) \\
& =S(r, w),
\end{aligned}
$$

and by (69),

$$
N\left(r, \frac{1}{\Delta w / w}\right)=T\left(r, \frac{\Delta w}{w}\right)+S(r, w) \geq T(r, w)+S(r, w) .
$$

Then $\lambda(\Delta w / w) \geq \sigma(w)$. So, $\lambda(\Delta w / w)=\sigma(w)$.

\section{Conflict of Interests}

The authors declare that there is no conflict of interests regarding the publication of this paper.

\section{Acknowledgment}

The project was supported by the National Natural Science Foundation of China (11171119). 


\section{References}

[1] L. Fuchs, "Sur quelques équations différentielles linéares du second ordre," Comptes Rendus de l'Académie des Sciences, vol. 141, pp. 555-558, 1905.

[2] B. Gambier, "Sur les équations différentielles du second ordre et du premier degré dont l'intégrale générale est a points critiques fixes," Acta Mathematica, vol. 33, no. 1, pp. 1-55, 1910.

[3] P. Painlevé, "Mémoire sur les équations différentielles dont l'intégrale générale est uniforme," Bulletin de la Société Mathématique de France, vol. 28, pp. 201-261, 1900.

[4] P. Painlevé, "Sur les équations différentielles du second ordre et d'ordre supérieur dont l'intégrale générale est uniforme," Acta Mathematica, vol. 25, no. 1, pp. 1-85, 1902.

[5] M. J. Ablowitz, R. Halburd, and B. Herbst, "On the extension of the Painlevé property to difference equations," Nonlinearity, vol. 13, no. 3, pp. 889-905, 2000.

[6] W. K. Hayman, Meromorphic Functions, Clarendon Press, Oxford, UK, 1964.

[7] L. Yang, Value Distribution Theory and Its New Research, Science Press, Beijing, China, 1982, (Chinese).

[8] R. G. Halburd and R. J. Korhonen, "Existence of finite-order meromorphic solutions as a detector of integrability in difference equations," Physica D: Nonlinear Phenomena, vol. 218, no. 2, pp. 191-203, 2006.

[9] Z.-X. Chen and K. H. Shon, "Value distribution of meromorphic solutions of certain difference Painlevé equations," Journal of Mathematical Analysis and Applications, vol. 364, no. 2, pp. 556566, 2010.

[10] O. Ronkainen, "Meromorphic solutions of difference Painlevé equations," Annales Academiae Scientiarum Fennicae, vol. 155, p. $59,2010$.

[11] J. L. Zhang and L. Z. Yang, "Meromorphic solutions of Painlevé III difference equations," Acta Mathematica Sinica, vol. 57, no. 1, pp. 181-188, 2014 (Chinese).

[12] S.-T. Lan and Z.-X. Chen, "On properties of meromorphic solutions of certain difference painlevé III equations," Abstract and Applied Analysis, vol. 2014, Article ID 208701, 9 pages, 2014.

[13] Y.-M. Chiang and S.-J. Feng, "On the Nevanlinna characteristic of $f(z+\eta)$ and difference equations in the complex plane," Ramanujan Journal, vol. 16, no. 1, pp. 105-129, 2008.

[14] R. G. Halburd and R. J. Korhonen, "Difference analogue of the lemma on the logarithmic derivative with applications to difference equations," Journal of Mathematical Analysis and Applications, vol. 314, no. 2, pp. 477-487, 2006.

[15] I. Laine and C.-C. Yang, "Clunie theorems for difference and $q$-difference polynomials," Journal of the London Mathematical Society, vol. 76, no. 3, pp. 556-566, 2007. 


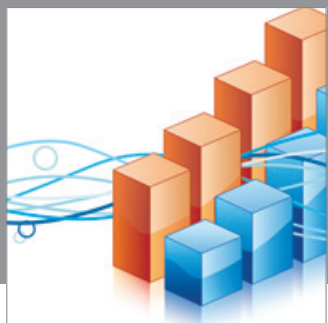

Advances in

Operations Research

mansans

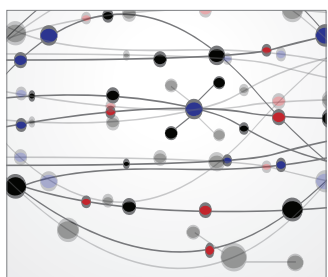

The Scientific World Journal
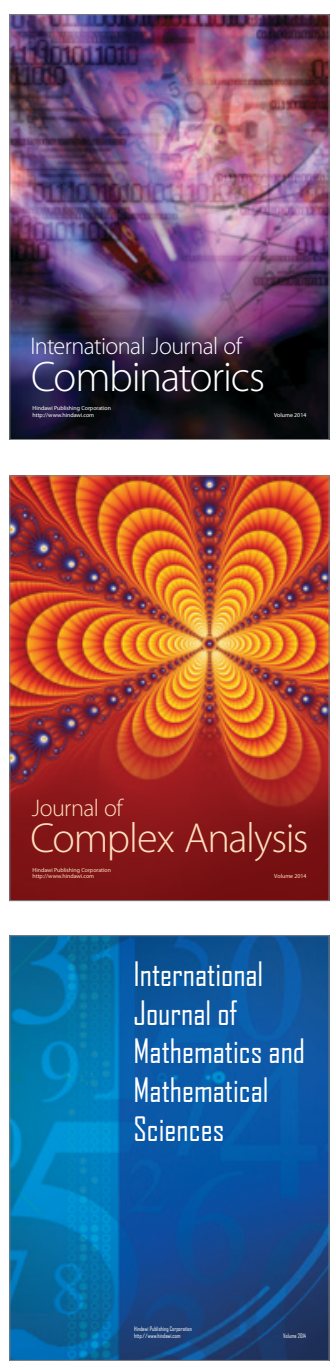
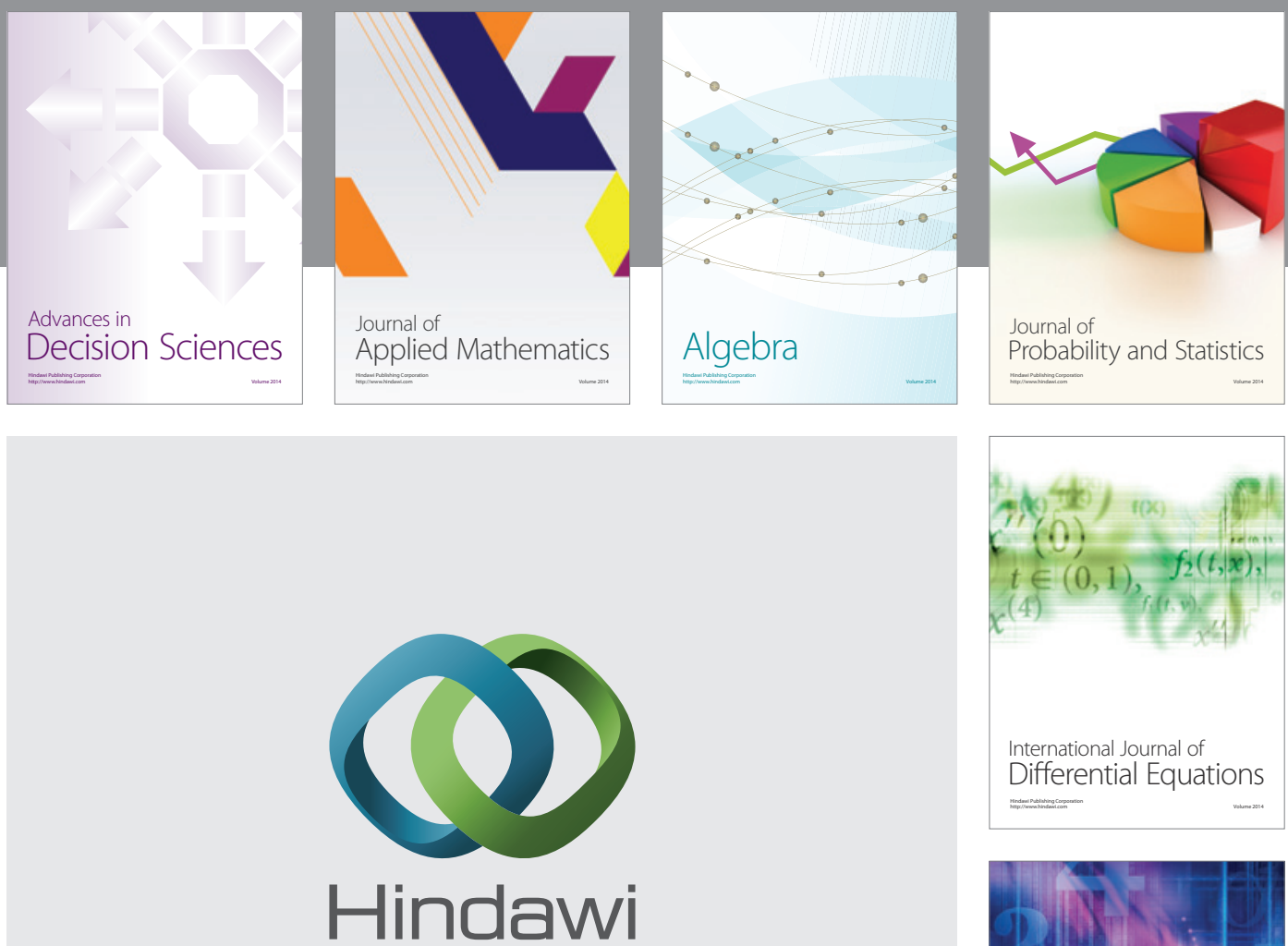

Submit your manuscripts at http://www.hindawi.com
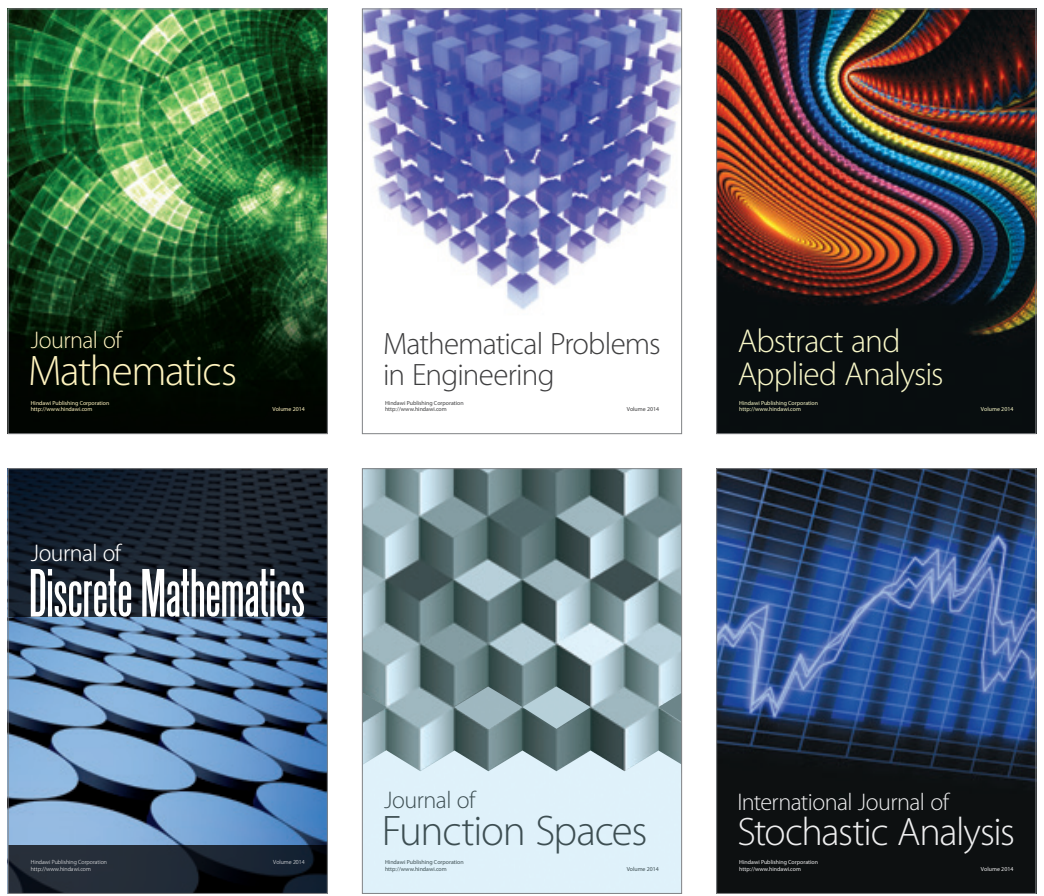

Journal of

Function Spaces

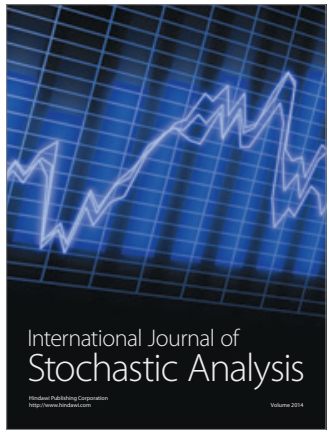

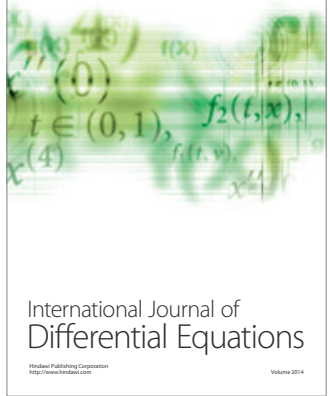
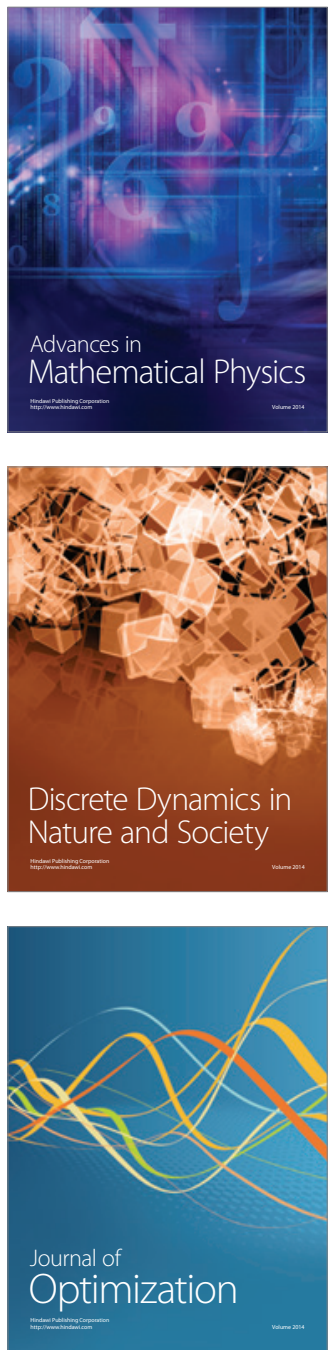